\title{
LA DISCIPLINARISATION DES SAVOIRS LINGUISTIQUES DANS L'ESPACE SCOLAIRE BRESILIEN : LE LOGICISME ET LE SOCIOLOGISME
}

\author{
THE DISCIPLINARIZATION OF LINGUISTIC \\ KNOWLEDGE IN THE BRAZILIAN SCHOOL SPACE: \\ LOGICISM AND SOCIOLOGISM
}

\author{
Mariza Vieira da Silva \\ Universidade Católica de Brasília, Brasília, Brasil
}

\begin{abstract}
Résumé: Le but de cet article est de comprendre comment le logicisme et le sociologisme se sont articulés dans la seconde moitié du XXe siècle, dans un contexte de redémocratisation, dans le discours de divulgation scientifique de la linguistique en tant qu'un nouveau champ disciplinaire pour l'enseignement du portugais, travaillant les éléments d'une contradiction toujours présente dans les théories: qu'il y a la langue et il y a des langues. L'Histoire des Idées Linguistiques et Analyse du Discours sont nos références théoriques et méthodologiques, ayant comme corpus deux livres publiés dans les années 1980, destinés, principalement, à la formation des enseignants, qui montrent comment se sont formées les alliances entre logicisme et sociologisme, soutenues par la pragmatique et par le fonctionnalisme, établissant des liens spécifiques entre l'universel et le particulier, créant des conditions de diffusion et de cristallisation de certaines oppositions et d'un technicisme d'instrumentalisation de la langue, effaçant ce qui lui est propre.
\end{abstract}

Mots-clés: Disciplinarisation du portugais; théories linguistiques; discours; sujet; sens.

Abstract: The aim of this paper is to understand how logicism and sociologism have articulated in Brazil, in the second half of the 20th century, in a context of redemocratization, in the discourse of scientific dissemination of Linguistics as a new disciplinary field of Portuguese teaching, dealing with the elements of a contradiction always present in the theories: that there is "the" language and there are "the" languages. Our theoretical and methodological references are the History of Linguistic Ideas and Discourse Analysis and we have as corpus two books published in the 1980s, which aim mainly at the training of teachers of basic education that stress how the alliances between logicism and sociologism were formed, supported by pragmatics and functionalism, establishing specific links between the universal and the particular, creating conditions for the diffusion and crystallization of certain oppositions and a technicism of instrumentalization of the language, erasing what is a property of that one

Keywords: Disciplinarization of Portuguese; linguistic theories; discourse; subject; meaning. 


\section{Introduction}

Pêcheux et Gadet (1977) dans l'article intitulé Y a-t-il une voie pour la linguistique hors $d u$ logicisme et $d u$ sociologisme, disent en rapport à la contradiction toujours présente entre l'unité construite historiquement et la diversité concrète des langues :

[...] selon nous, l'histoire même de la linguistique peut être comprise comme une sorte de "lutte entre deux voies " - le logicisme et le sociologisme formant les éléments d'une contradiction qui prend successivement de multiples formes, depuis la pré-histoire de la linguistique jusque dans ses aspects les plus modernes, les plus actuels et les plus scientifiques (PÊCHEUX; GADET, 1977, p. 133).

Le but de cet article est, ainsi, de comprendre comment le logicisme et le sociologisme se sont articulés dans la seconde moitié du XXe siècle, dans le discours de divulgation scientifique, destiné, principalement, à la formation des enseignants, dans une conjoncture de déclin de la dictature militaire, travaillant les éléments de cette contradiction, pour aider dans l'analyse du problème de la crise dans l'enseignement de la langue portugaise et celui de l'échec scolaire marqué par l'abandon et le redoublement, et, en même temps, des inégalités et des différences sociales et linguistiques qui deviennent de plus en plus visibles. Comprendre comment, dans ce processus d'articulation, le discours sur la langue, soutenu par les savoirs linguistiques et les institutions, normalise la langue ainsi que notre relation avec elle, produisant l'effet d'une langue unique et homogène, ce qui signifie saisir (apreender) les alliances qui se forment dans le travail de cette contradiction.

Ces compréhensions impliquent de prendre l'éthique linguistique du point de vue non seulement des résultats, mais des présupposés de travail de la et sur la langue

O saber metalinguístico que produzimos, e que deve estar à disposiçáo de todos na sociedade, já traz inscritas suas direçôes e consequências no momento mesmo em que o formulamos. E sua formulação [e transmissão, acrescentaríamos] deriva do lugar (teórico-científico) em que ele se constitui, produzindo efeitos sobre os sentidos dos objetos que ele produz (ORLANDI, 1998 , p. 15$)^{1}$.

1 Le savoir métalinguistique que nous produisons, et qui devrait être disponible à tous 
Nos références théoriques et méthodologiques pour l'élaboration de ce travail ont été l'Histoire des Idées Linguistiques et l'Analyse de Discours, dont l'objet, le discours, est défini comme " effets de sens entre locuteurs "(PÊCHEUX, 1969). Nous avons sélectionné comme corpus deux livres publiés dans les années 1980, avec des éditions successives, dans une série intitulée "Fundamentos", écrits par des auteures d'expertise reconnue dans et hors de la communauté scientifique, appelés : "No mundo da escrita: uma perspectiva psicolinguística " et " Linguagem e escola: uma perspectiva social » de Mary A. Kato (1986) et Magda Soares (1986), respectivement. Ce sont des livres que nous pouvons considérer de divulgation de la linguistique comme (un) nouveau champ disciplinaire pour l'enseignement du portugais et qui aident à construire la pensée pédagogique brésilienne à un moment historique déterminé ; pensée encore dominante, certains déplacements étant considérés. Donc, nous ne visons pas à étudier les positions théoriques et politiques de Soares et Kato pour eux-mêmes.

Cet article fait partie d'un ensemble plus large d'études et de recherches, que nous avons développé sur la constitution de la discipline scolaire des Portugais à partir de la seconde moitié du XXe siècle, ayant les théories linguistiques comme savoirs de référence, et construisant une vaste archive formée par différentes matérialités discursives, et où nous cherchons à comprendre les rapports entre l'histoire conceptuelle et l'histoire institutionnelle, à penser l'historicité de ces rapports dans des contextes historiquement déterminés.

\section{Conjoncture historique et théorique}

Les livres analysés ont été publiés, comme nous le disons, dans les années 1980, dans un contexte de redémocratisation du pays après vingt et un ans de dictature, dans un moment historique où un nouveau modèle de société lié à la démocratie commence à prendre forme. À ce moment-là, la question sociale, la question éducative et la question linguistique sont remises en question, analysées et des solutions sont proposées, en déplaçant le problème de l'inégalité de classe sociale vers celui des inégalités scolaires. En rapport à la langue à enseigner à l'école, la contradiction entre l'unité et la diversité est explicitement manifestée dans les textes officiels comme dans la présentation d'Annales d'un événement organisé par l'Instituto Nacional de Pesquisas Educacionais - INEP, comme un moyen de produire des déplacements dans la configuration du portugais en tant que langue

dans la société, trouve déjà inscrites ses directions et ses conséquences au moment où nous le formulons. Et sa formulation [et la transmission, ajouteraient] dérive de la place (théorique et scientifique) où il se constitue, produisant des effets sur les sens des objets qu'il produit (ORLANDI, 1998, p. 15). 
nationale.

[...] as questōes versavam não só sobre preocupaçōes com novas pedagogias ou didáticas da aprendizagem da língua, em seu uso oral e escrito, envolvendo propostas para a produção de materiais instrucionais, mas também sobre a dialética da diferenciação-unificação da língua (BRASIL, 1983, p. 7)².

Une autre question qui marquera de façon décisive ce processus de redémocratisation de la société en tant que contexte de changements dans l'éducation, est l'urbanisation croissante et désordonnée, donnant une visibilité aux inégalités et aux différences individuelles et régionales. La relation ville-campagne est présente dans les politiques publiques et dans les études de la sociolinguistique variationnelle, ayant pour centre de référence la ville. O III Plano Setorial de Educação, Cultura e Desporto : 1980-1985 affirme "sua absorvente preocupação com as áreas mais carentes, precisamente o campo e a margem urbana» (BRASIL, 1980, p. $9)^{3}$. Il présente comme lignes prioritaires nationales : l'éducation dans les zones rurales, l'éducation dans les périphéries urbaines, le développement culturel et la valorisation des ressources humaines.

À partir des années 1970, le ministère de l'Éducation avait déjà commencé à travailler en collaboration avec la communauté scientifique pour la production des politiques, des projets, des programmes, de la législation, en donnant aux propositions de l'État le soutien explicite de la science. Cette collaboration s'est intensifiée et renforcée dans les années 1980. Cela a amené des possibilités pour que les théories linguistiques soient nationalement connues et que les affrontements et les alliances entre elles se mettent en mouvement. Dans les " Parâmetros Curriculares Nacionais» des années 1990, qui font partie des politiques de l'État, nous trouvons les affirmations qui suivent :

A nova crítica do ensino de Língua Portuguesa, no entanto, só se estabeleceria mais consistentemente no início dos anos 80 , quando as pesquisas produzidas por uma linguística independente da tradição normativa e filológica e os estudos desenvolvidos em variação linguística e

$2[\ldots]$ les questions traitaient non seulement les préoccupations pour de nouvelles pédagogies ou didactiques d"apprentissage de la langue, dans leur usage oral et écrit, impliquant des propositions pour la production de matériel pédagogique, mais aussi la dialectique de la différenciation-unification de la langue (BRASIL, 1983, p. 7).

3 "sa préoccupation absorbante pour les zones les plus défavorisées, précisément la campagne et la marge urbaine » (BRASIL, 1980, p. 9). 
psicolinguística, entre outras, possibilitaram avanços nas áreas de educação e, psicologia da aprendizagem, principalmente no que se refere à aquisição da escrita (BRASIL, 1998, p. 17) $)^{4}$.

À côté de cette action politique vis-à-vis des pratiques pédagogiques, nous avons ce que Chiss et Puech (1999) appellent « effets de retour » sur le domaine scientifique par la proposition de thèmes de recherche, thèses, projets et par le financement de recherches, ainsi que par la formation d'une langue imaginaire en rapport à la/aux langue(s) du Brésil et à ses parleurs. Les linguistes occupent, ainsi, une position de critiques à l'enseignement conservateur, normatif, discriminatoire de la langue et, en même temps, une position de participants de la formulation des politiques, de l'élaboration des lois, des manuels, qui traitent de la langue et de son enseignement de façon novatrice, descriptive et inclusive, aussi bien que proposant des cours et des programmes de formation des enseignants. Ils occupent, donc, une position sujet paradoxale et opaque, engagée consciemment ou non dans un projet politique de l'État.

\section{L'éducation des couches populaires \& la formation d'individus fonctionnellement lettrés}

Ce sont les deux objectifs principaux des livres analysés dans le contexte plus large de l'universalisation de l' éducation fondamentale - 7 à 14 ans - en tant que droit de tous et devoir de l'État. Selon Soares, d'après le recensement de 1980, plus de 30\% des Brésiliens entre 7 à 14 ans étaient hors du système scolaire, chiffre arrivant à $50 \%$ dans certains États. Nous y trouvons certains éléments présents jusqu'à aujourd'hui dans le processus de disciplinarisation scolaire du portugais, même sous une forme transformée, déplacée, cherchant à surmonter les contradictions historiques d'une société esclavagiste, structurellement inégale.

Voici deux premières séquences linguistico-discursives - SLD -, afin que nous puissions montrer de manière plus large les hypothèses qui y sont travaillées. D’abord, nous présentons le point de vue sociologique.

\section{SLD 1}

Nossa escola tem-se mostrado incompetente para a educação das camadas

4 "sa préoccupation absorbante pour les zones les plus défavorisées, précisément la campagne et la marge urbaine" (BRASIL, 1980, p. 9). 
populares, e essa incompetência, gerando o fracasso escolar, tem tido o grave efeito não só de acentuar as desigualdades sociais, mas, sobretudo, de legitimá-las.

Grande parte da responsabilidade por essa incompetência deve ser atribuída a problemas de linguagem: o conflito entre a linguagem de uma escola fundamentalmente a serviço das classes privilegiadas, cujos padrōes linguísticos usa e quer ver usados, e a linguagem das classes populares, que essa escola censura e estigmatiza, é uma das principais causas do fracasso dos alunos pertencentes a essas camadas, na aquisição do saber escolar (SOARES, 1986, p. 6) ${ }^{5}$.

Nous soulignons dans cette séquence initiale, certains énoncés qui seront réitérés, paraphrasés, dans le livre de Soares: la notion de compétence comme principale directrice du travail pédagogique; l'échec scolaire comme produit de l'incompétence; les problèmes de langage comme responsables de cette incompétence ; les classes sociales - privilégiées et populaires traitées de manière dichotomique, dans une opposition linéaire ainsi que les langues qu'elles parlent.

La représentante du logicisme, de l'autre côté, se concentre directement sur la question du sens, du sujet et de son comportement, travaillant le rapport entre langage et cognition du point de vue de la communication.

\begin{abstract}
SLD 2
Meu pressuposto, neste livro, é o de que a função da escola na área de linguagem é introduzir a criança no mundo da escrita, tornando-a um cidadão funcionalmente letrado, isto é, um sujeito capaz de fazer uso da linguagem escrita para sua necessidade individual de crescer cognitivamente e para atender às demandas de uma sociedade que prestigia esse tipo de linguagem como um dos instrumentos de comunicação (KATO, 1986, p. 7)..
\end{abstract}

$5 \quad$ Notre école s'est montrée incompétente pour l'éducation des couches populaires, et cette incompétence, générant l'échec scolaire a eu le grave effet non seulement d'accentuer les inégalités sociales, mais surtout de les légitimer. Une grande partie de la responsabilité de cette incompétence doit être attribuée à des problèmes linguistiques : le conflit entre le langage d'une école fondamentalement au service des classes privilégiées, dont elle utilise les modèles linguistiques et qu'elle veut voir utilisés, et le langage des classes populaires, que cette école censure et stigmatise, est l'une des principales causes de l'échec des étudiants appartenant à ces couches, dans l'acquisition du savoir scolaire (SOARES, 1986, p. 6)

6 Ma présupposition de ce livre est que la fonction de l'école dans le domaine du langage, est d'introduire l'enfant dans le monde de l'écriture, ce qui le fait un citoyen fonctionnellement lettré, c'est-à-dire, un sujet capable d'utiliser le langage écrit pour ses nécessités individuelles de croître cognitivement et pour répondre aux demandes d'une société qui honore ce type de langage comme l'un des outils de communication (KATO, 1986, p. 7). 
Les chapitres du livre travailleront cette présupposition répondant à trois questions de l'auteur, visant à la formation des enseignants : 1. Quelle est la nature formelle et fonctionnelle de l'écriture ? 2. Que faisons-nous lorsque nous lisons et écrivons ? 3. Comment pouvons-nous apprendre à lire et à écrire?

Suivant des chemins propres au logicisme et au sociologisme, ces matérialités linguistico-discursives aident à construire un cadre théoricopolitique propre, qui n'est pas une simple reproduction ou transposition du discours savant. Ce cadre s'installe dans différentes discursivités comme évidences, ce qui contribue à la production d'un consensus comme produit historique structuré et structurant des pratiques pédagogiques. Il est important de noter que ce consensus, la conciliation toujours présente dans l'histoire de l'éducation du Brésil dont parle Cury, n'est pas homogène. «Em síntese, a conciliação é a aliança dos mesmos, o silenciamento dos contrários e a exclusão dos radicalmente outros, isto é, dos contraditórios " (CURY, 2001, p. 20) ${ }^{7}$.

En ce sens, nous avons sélectionné pour cet article, certains points présents dans les formulations de ces deux auteures, qui signalent la façon dont le sociologisme et le logicisme ont été institutionnalisés à l'école comme approches théoriques complémentaires, de même qu'ils montrent les tendances qui ont soutenu les alliances entre le sociologisme et le logicisme, afin de créer les conditions d'une telle conciliation : la Pragmatique et le Fonctionnalisme. Ce sont des points communs, mais pas homogènes, ni serrés, et ne peuvent pas être pris de manière positive. Ils se nourrissent, renforçant certaines affiliations, certains sens et certains processus de subjectivation construits à partir du sujet en situation et en action et qui vont vers un sujet universel, situé partout et nulle part (PÊCHEUX, 1975). Ces points sont : l'interdisciplinarité ; l'argument de scientificité ; la notion de l'histoire; la notion de communication; les notions de langage et langue.

L'interdisciplinarité est traitée comme moyen de construire un objet total qui peut être transmis, enseigné, appris. Soares vise à articuler et intégrer les théories originaires de la Sociologie, de la Sociologie du Langage et de la Sociolinguistique afin de comprendre " le caráter político-ideológico do uso e do ensino da língua na escola » (SOARES, 1986, p. 7), « ao lado da também indispensável perspectiva psicolinguística "(SOARES, 1986, p.6) ${ }^{8}$.

7 " En synthèse, la conciliation est l'alliance du même, le taire des contraires et l'exclusion radicalement d'autres, c'est-à-dire, contradicteurs " (CURY, 2001, p. 20).

8 " caractère politico-idéologique de l'usage et de l'enseignement de la langue à l'école " (SOARES, 1986, p. 7), "à côté de l'également indispensable perspective psycholinguistique " 
L'accent est mis sur les questions linguistiques, à travers des auteurs tels que Bernstein, Labov, Bourdieu, Sapir et Whorf, Piaget, Vygotsky et Luria. Par le logicisme, cette avancée interdisciplinaire devient moins présente dans le livre, en cherchant à préserver l'autonomie relative de la Linguistique; et en apportant d'autres domaines de la Linguistique pour la compréhension du problème.

\begin{abstract}
SLD 4
Para ter um quadro mais completo do fenômeno da aprendizagem linguística, o leitor deverá examinar obras de cunho sociolinguístico, em que variáveis como classe social, dialetos regionais, normas gramaticais e padrôes de uso são detalhadamente discutidas. Seria desejável ainda um maior aprofundamento nas seguintes áreas: a\} linguística textual, b) análise conversacional, c) psicologia cognitiva (para aprofundamento das questóes relativas à memória) e d) aquisiçấo da linguagem (KATO, 1986, p. 9) ${ }^{9}$.
\end{abstract}

L'argument descientificité est toujours présent comme effet idéologique, comme une manière de faire face à un enseignement de la langue considéré conservateur et non démocratique soutenu par la philologie et la grammaire appelée "traditionnelle". Dans le sociologisme, il se montre dans un discours engagé politiquement, rhétorique, soutenu par des oppositions, en parlant de questions sociales et politiques et, dans le même mouvement, en cachant les conflits et les contradictions qui y sont présents.

Attire notre attention le nom donné aux théories : celui d' « idéologie " non conceptualisée, mais traitée tout au long du livre, comme contenu, comme vision du monde, commandant les modes d'existence des théories et de la validation de celles-ci. Nous pensons que ce serait une façon d'organiser le rapport de la Linguistique avec les Sciences Humaines et les Sociales, à un moment où il faut parler de l'inégalité, de démocratisation, de rapports de production, de capitalisme, de questions sociales et politiques corrélationnelles à l'éducation ; une façon d'organiser le rapport à la/aux langue(s) et son enseignement avec le contexte historique, la situation, le

(SOARES, 1986, p. 6).

9 Pour avoir un cadre plus complet du phénomène de l'apprentissage linguistique, le lecteur devra examiner des œuvres de nature sociolinguistique, où des variables telles que la classe sociale, les dialectes régionaux, les normes grammaticales et standards d'usage sont discutés en détail. Un approfondissement serait en outre souhaitable dans les domaines suivants: a) la linguistique textuelle, b) l'analyse conversationnelle, c) la psychologie cognitive (pour un examen plus approfondi des questions liées à la mémoire) et d) l'acquisition du langage (KATO, 1986, p. 9). 
sujet, faisant une lecture fonctionnaliste de la conjoncture historique et des théories.

SLD 5

A ideologia do dom oculta-se sob um discurso que se pretende científico" [contudo] "a cientificidade de seus pressupostos foi irremediavelmente abalada quando se evidenciou a partir da ampliação do acesso das camadas populares à escola, que as "diferenças naturais" nấo ocorriam, na verdade, apenas entre indivíduos, mas, sobretudo, entre grupos de indivíduos entre os grupos social e economicamente privilegiados e os grupos desfavorecidos, entre pobre e ricos, entre as classes dominantes e as classes dominadas (SOARES, 1986, p. 11 - italique ajouté par l'auteur) ${ }^{10}$.

L'argument de scientificité est également présent dans le livre de Kato, mais marqué par le fonctionnement plus proche du discours scientifique. Le texte est produit comme un compte rendu bibliographique présentant des résultats d'études et de recherches, soutenus par l'autorité des nombreux auteurs cités, la plupart étrangers, liés en grande partie à New Literacy Studies, par une terminologie et des notions du domaine de la Linguistique et en même temps, par une profusion de schémas, tableaux, figures, exemples souvent créés par l'auteur. À la fin de chaque section et de chaque chapitre sont présentés des résumés et des conclusions, respectivement, rendant la lecture plus accessible à un public non spécialiste, préfigurant les affiliations de l'auteure et les alliances entre les théories.

L'histoire des sciences et l'histoire de la lecture et de l'écriture sont traitées de façon linéaire, évolutive, en cherchant une conciliation entre les termes et les notions, par le fonctionnement de la " philosophie spontanée " (PÊCHEUX; GADET, 1977), en recouvrant les contradictions du champ de la Linguistique et du champ de l'Éducation.

SLD 6

As teorias discutidas nos capítulos anteriores, embora contraditórias em vários aspectos, levam, necessariamente - em decorrência daquilo que é

10 L'idéologie du don se cache dans un discours destiné à être "scientifique " [mais] " la scientificité de ses présupposés a été irrémédiablement brisée quand il est mis en évidence, à partir de l'accroissement de l'accès des couches populaires à l'école, que les "différences naturelles " n'ont pas eu lieu en fait, seulement chez les individus, mais surtout parmi les groupes d'individus, parmi les groupes socialement et économiquement privilégiés et les groupes défavorisés, entre pauvres et riches, entre les classes dominantes et les classes dominées (SOARES, 1986, p. 11). 
comum entre elas e verdadeiro em cada uma - a uma mesma conclusão: as relaçôes entre linguagem e classe social têm forçosamente, de estar presentes, numa escola transformador, na definiçấo dos objetivos do ensino da língua materna, na seleção e organização do conteúdo, na escolha de métodos e procedimentos de ensino e na determinação de critérios de avaliação da aprendizagem (SOARES, 1986, p. 76-77) ${ }^{11}$.

Pour la psycholinguistique cognitive, l'histoire ne sert même pas comme corrélation pour expliquer les différences sociales et individuelles.

\begin{abstract}
SLD 7
3. Há variação na forma pela qual as atividades linguísticas são distribuídas entre as duas modalidades [oral e escrita], variaçáo dependente da evolução histórica e, sincronicamente, das diferenças sociais, principalmente funcionais, havendo, além disso, variaçâo individual, que independe de variáveis externas (KATO, 1986, p. 41) ${ }^{12}$.
\end{abstract}

La communication comme cadre théorico-idéologique logera les termes et les notions dans la recherche d'une communication univoque, sans équivoque, dans les rapports sociaux et politiques, dans les pratiques pédagogiques. Ce cadre large est construit au Brésil de façon décisive dans les années 1970 avec l'articulation de différentes discursivités : juridiques, politiques, administratives, académiques, didactiques (cf. SILVA, 2017; 2012), faisant circuler et légitimer certaines notions. Selon Kato (1986, p. 97), "Ler e escrever são atos de comunicação em que um dos parceiros é apenas imaginado, representado. Como atos de comunicação, estão sujeitos a todos os princípios que regem a comunidade verbal oral " (KATO, 1998, p. 97$)^{13}$.

11 Les théories discutées dans les chapitres précédents, bien que contradictoires à bien des égards, conduisent nécessairement - à la suite de ce qui est commun entre eux et vrai dans chacun - à la même conclusion : les rapports entre le langage et la classe sociale doivent nécessairement être présents, dans une école transformatrice, dans la définition des objectifs d'enseignement de la langue maternelle, dans la sélection et l'organisation du contenu, dans le choix des méthodes et des procédures d'enseignement et dans la détermination des critères d'évaluation des apprentissages (SOARES, 1986, p. 76-77).

12 Il existe une variation dans la façon dont les activités linguistiques sont distribuées entre les deux modalités [orale et écrite], variation qui dépend de l'évolution historique et, synchroniquement, des différences sociales, principalement, fonctionnelles, ayant, en plus, la variation individuelle, qui est indépendante des variables externes (KATO, 1986, p. 41).

13 "Lire et écrire sont des actes de communication dans lesquels un des partenaires est seulement imaginé, représenté. Comme actes de communication, ils sont soumis à tous les 
La langue et le langage sont traités comme notions interchangeables, la notion de «langage» étant prédominante dans les deux tendances. Dans le sociologisme, l'objet "langue " n'est pas défini, décrit par ce qui lui est propre, mais comme constitué par des influences extérieures, par la structure de la société comme une corrélation. Il est important de noter que cette indistinction théorique permet de comprendre, dans l'analyse d'autres discours, l'absorption de la langue dans l'ensemble plus large des «langages», ayant pour effet effacer, taire le réel de la langue et le réel de l'histoire (GADET; PÊCHEUX, 1981).

\section{La Pragmatique et le Fonctionnalisme}

En considérant les découpes analysées jusqu'ici, nous réitérons que la lecture que l'on fait du sociologisme et du logicisme, comme une dualité contradictoire qui se matérialise dans la structure des théories linguistiques et de leurs relations, est soutenue par la Pragmatique et par le Fonctionnalisme. Ils permettent d'établir des liens entre les particularismes culturels, les variétés, les usages, les compétences communicatives et les universalismes de la compétence cognitive, de la constitution d'un sujet naturel-logique, établissant une relation entre une structure logique et une situation observable.

La Pragmatique travaille la langue en rapport à l'extériorité. C'est un champ disciplinaire soumis à des luttes internes et externes, peut-être, par la multitude de sens donnés au terme, disent Ducrot \& Schaeffer (1995) :

La pragmatique étudie tout ce qui, dans le sens d'un énoncé, tient à la situation dans laquelle l'énoncé est employé, et non à la seule structure linguistique de la phrase utilisée (DUCROT; SCHAEFFER, 1995, p. 131). $[\ldots]$

La pragmatique concerne non pas l'effet de la situation sur la parole, mais celui de la parole sur la situation. La plupart de nos énoncés, en même temps qu'ils donnent des renseignements sur le monde, instaurent, ou prétendent instaurer, entre les participants au discours, un type particulier de rapports selon l'acte de langage accompli selon, par exemple qu'il s'agit d'une interrogation ou d'un ordre), selon aussi le niveau de discours choisi (selon si la parole est déférente ou familière) (DUCROT; SCHAEFFER, 1995, p. 133).

Elle traite, donc, de la situation, des actes delangage, de la représentation

principes qui régissent la communauté verbale orale " (KATO, 1986, p. 97). 
d'un sujet intentionnel - un usager - qui prend le langage comme action et intervient en tant qu'agent de changements sociaux dans une société où les incompréhensions, les conflits sont provoqués essentiellement par des problèmes de communication. En étudiant l'utilisation du langage dans les activités humaines, les usages acquièrent un rôle central, marqué, maintes fois, par des frontières stables, ignorant la complexité des relations sociales (urbaines ou non) dans lesquelles de nouvelles références, de nouvelles relations de sens et de force émergent, extrapolant les dichotomies déjà établies.

\begin{abstract}
SLD 8
É o uso da língua na escola que evidencia mais claramente as diferenças entre grupos sociais e que gera discriminaçóes e fracasso: o uso, pelos alunos provenientes das camadas populares, de variantes linguísticas social e escolarmente estigmatizadas provoca preconceitos linguísticos, e leva a dificuldades de aprendizagem, já que a escola usa e quer ver usada a variantepadrão socialmente prestigiada (SOARES, 1986, p. 17) ${ }^{14}$.
\end{abstract}

Même la crise de la langue est définie par Soares comme «um uso inadequado e deficiente da língua materna e como uma decadência de seu ensino " étant " a escola é que vem gerando o conflito, a crise, que é o resultado de transformaçóes quantitativas - maior número de alunos - e, sobretudo qualitativas - distância cultural e linguística entre os alunos... (SOARES, 1986, p. 68 - italique ajouté par l'auteur)» ${ }^{15}$.

Quant au logicisme, dans le Vocabulaire Critique, qui est à la fin du livre de Kato, nous avons la Pragmatique définie comme « estudos dos significados em contexto, levando-se em conta os usuários das expressóes linguísticas » (KATO, 1986 , p. 141). Dans ce Vocabulaire, les usages, en particulier, seront attribués à la définition du Fonctionnalisme «abordagem que enfatiza o uso mais do que a forma ou que parte do uso para a forma

14 C'est l'usage de la langue à l'école qui montre plus clairement les différences entre les groupes sociaux et qui génère les discriminations et l'échec : l'usage par les élèves des classes populaires, de variations linguistiques socialement et scolairement stigmatisées provoque des préjugés linguistiques, et conduit à des difficultés d'apprentissage, puisque l'école utilise et veut voir utilisée la variation-standard socialement prestigieuse (SOARES, 1986, p. 17).

15 «... un usage inadéquat et déficient de la langue maternelle et comme une décadence de son enseignement " étant "l'école est que génère le conflit, la crise, celle qui est le résultat des transformations quantitatives - plus d'étudiants - et surtout qualitatives - distance culturelle et linguistique entre les étudiants...» (SOARES, 1986, p. 68 - italique ajouté par l'auteur). 
"(KATO, 1986, p. 140 $)^{16}$. L'accent mis sur le travail avec les usages est également présent dans son œuvre, comme les sous-titres de la troisième section intitulée "La parole et l'écriture: différences fonctionnelles".

\author{
SLD 9 \\ O uso da escrita na história do homem \\ Os usos da escrita em uma sociedade letrada \\ O uso da escrita em nosso contexto ${ }^{17}$
}

Le Fonctionnalisme, selon Auroux \& Weil (1991, p. 150), « est une conception épistémologique selon laquelle l'explication d'une certaine classe de phénomènes repose sur leur fonction plutôt que sur leur structure intrinsèque et les processus qui en découlent ". Pour les auteurs, ce fut surtout dans les sciences humaines et sociales que le Fonctionnalisme a gagné en force, spécialement dans le domaine de la Sociologie entre les années 1920 et 1960; et ils considèrent que l'un des plus importants de ses problèmes en termes épistémologiques,

[...] réside dans la nécessité où il se trouve d'élaborer une liste de fonctions claires et susceptibles d'une définition indépendante. On remarquera qu'il s'agit souvent d'éléments extrêmement vagues et d'une grande trivialité. Ainsi en est-il des quatre impératifs, qui, selon Parsons, permettent à un système social d'exister et de se maintenir : - la poursuite de buts ; - l'adaptation au milieu environnant ; - l'intégration des membres dans le système social ; - la stabilité normative (AUROUX; WEIL, 1991, p. 151).

Ces impératifs qui sont, nous ajoutons, en étroite relation avec les demandes économiques, sociales et politiques qui soutiennent les pratiques linguistico-pédagogiques développées à l'école comme une institution de l'État brésilien, dans une société marquée par l'inégalité sociale profonde et par l'hétérogénéité linguistique et discursive.

Le Fonctionnalisme a la description comme une pratique scientifique centrale en opposition au prescriptivisme et vise à prendre en compte les fonctions exercées par les éléments linguistiques. Nous savons que la notion

16 "... d'études des significations en contexte, en tenant compte des utilisateurs des expressions linguistiques" (KATO, 1986, p. 141) [...] approche qui met l'accent sur l'usage plus que la forme ou qui part de l'usage à la forme " (KATO, 1986, p. 140).

17 L'usage de l'écriture dans l'histoire de l'homme / L'usage de l'écriture dans une société lettrée / L'usage de l'écriture dans notre contexte (KATO, 1986). 
de fonction peut être comprise de plusieurs manières ; et en ce sens, on peut dire qu' il y a plus d'un Fonctionnalisme.

Dans les archives analysées, la fonction et la forme constituent le noyau de ce cadre théorico-idéologique initialement mentionné. Pour les cognitivistes " $\mathrm{O}$ interesse dessa visão funcionalista reside no fato de que as formas novas que aparecem são justificadas em função das necessidades comunicacionais, o que permite examinar a relação entre forma e função " $\left(\right.$ KATO, 1986, p. 105) ${ }^{18}$. Le sociologisme va dans cette direction en disant que la théorie des différences linguistiques (LABOV) et la théorie du capital linguistique (BOURDIEU) «fundamentam-se num estudo descritivo das diferenças de linguagem entre classes sociais "(SOARES, 1986, p. 67) ${ }^{19}$. Déjà la théorie de la déficience linguistique

\begin{abstract}
SLD 11
[...] diverge das outras duas por assumir uma atitude prescritiva diante das diferenças de linguagem entre classes sociais; às diferenças, chama deficiências, e propóe-se corrigi-las. Essa atitude prescritiva, que se origina de uma postura valorativa diante de variedades linguísticas, é cientificamente inadmissível, para a Sociolinguística e a Sociologia contemporâneas; por isso, a proposta educacional que dela decorre - a educação compensatória é hoje inaceitável, dada a falsidade de seus pressupostos (SOARES, 1986, p. 76 - italique ajouté par l’auteure $)^{20}$.
\end{abstract}

Cependant, les choses ne sont pas aussi simples. Un critère d'évaluation est toujours présent.

\title{
SLD 12
}

É verdade que algumas línguas são funcionalmente mais desenvolvidas que outras: o inglês, por exemplo, é, atualmente, uma língua internacional,

18 «L'intérêt de ce point de vue fonctionnaliste réside dans le fait que les nouvelles formes qui apparaissent sont justifiées en fonction des nécessités de communication, ce qui ? permet d'examiner le rapport entre forme et fonction " (KATO, 1986, p. 105).

19 " ils sont basés sur une étude descriptive des différences de langage entre les classes sociales" (SOARES, 1986, p. 67).

$20[\ldots]$ differe des deux autres pour assumer une attitude prescriptive devant des différences de langage entre les classes sociales; à des différences, elle appelle déficiences, et se propose de les corriger. Cette attitude prescriptive, qui vient d'une position évaluative sur les variétés linguistiques, est scientifiquement inadmissible pour la Sociolinguistique et la Sociologie contemporaines; par conséquent, la proposition éducative qui en découle - l'éducation compensatoire - est maintenant inacceptable, compte tenu de la fausseté de ses présupposés (SOARES, 1986, p. 76). 
enquanto o português não o é; por outro lado, inglês, português, francês, alemão e várias outras línguas têm um sistema de escrita altamente sofisticado, que permite que sejam usadas tanto para uma conversaçáo casual quanto para a redação de artigos científicos, enquanto outras línguas não têm um sistema de escrita e, portanto, não podem exercer essa mesma variedade de funçôes (SOARES, 1986, p. 39 - italique ajouté par l'auteure) ${ }^{21}$.

En traitant de l'écriture, de ce que nous faisons lorsque nous écrivons, Mary Kato montre comment le Fonctionnalisme est lié à la gestion des politiques et des pratiques scolaires, en traitant en détail de la planification comme stratégie de la pratique scolaire, c'est-à-dire, du lieu du technicisme dans le processus de disciplinarisation de la langue portugaise, ce qui deviendra de plus en plus présent dans les politiques publiques à travers, principalement, les évaluations nationales et internationales développées dans les décennies suivantes.

\section{SLD 14}

Seguindo a mesma tendência funcionalista que está havendo hoje na linguística, os estudos sobre a produção oral e a escrita começam a ver essas atividades como açóes deliberadas, determinadas por um planejamento geral (KATO, 1986, p. 78) ${ }^{22}$.

\section{Pour conclure}

L'analyse nous a permis d'observer comment se sont construits des rapports entre deux temporalités : l'histoire des idées linguistiques et celle de la scolarisation du portugais dans une conjoncture théorique et politique donnée d'un pays capitaliste périphérique, analysant la manière dont le rationalisme et l'empirisme travaillent la contradiction omniprésente dans l'objet de la Linguistique qu'il y a de le langue et qu'il y a des langues,

21 Il est vrai que certaines langues sont fonctionnellement plus développées que d'autres: l'anglais, par exemple, est actuellement une langue internationale, alors que le portugais ne l'est pas; d'autre part, l'anglais, le portugais, le français, l'allemand et plusieurs autres langues ont un système d'écriture très sophistiqué, ce qui leur permet qu'elles soient utilisées à la fois pour une conversation casuelle et pour la rédaction d'articles scientifiques, alors que d'autres langues ne disposent pas d'un système d'écriture et par conséquent, elles ne peuvent exercer cette même variété de fonctions (SOARES, 1986, p. 39 - italique ajouté par l'auteure).

22 En suivant la même tendance fonctionnaliste qu'il y a aujourd'hui en linguistique, les études sur la production orale et l'écrit commencent à voir ces activités comme des actions délibérées, déterminées par une planification générale (KATO, 1986, p. 78). 
remettant en question le réel de la langue et le réel de l'histoire.

Observons qu'on va de la " que a função da escola, na área de linguagem, é introduzir a criança no mundo da escrita, tornando-a um cidadão funcionalmente letrado ${ }^{23}$ " vers un langage réduit à un cognitivisme inné (KATO, 1986, p. 7), «), à la compréhension du " caráter políticoideológico do uso e do ensino da língua na escola " ${ }^{24}$ (SOARES, 1986, p. 7), comme solution de compromis, mettant en évidence les implications épistémologiques, mais aussi sociales dans les pratiques scolaires : en cachant le symbolique et le politique du langage et des langues au réduire les langues à leur statut d'instrument soit de la communication, soit de la pensée; en établissant des liens particuliers entre le particularisme et l'universalisme que, paradoxalement, serait un moyen de construire la diversité; en absorbant les différences pour universaliser les rapports de travail et des droits.

Dans le sociologisme, les questions sociales et linguistiques sont traitées de façon généralisante, dans un discours sur les "marginalisés ", les "déficients», les « différents ", les «stigmatisés ", les " couches populaires ", les "dominateurs ", les « dominés ", en construisant des séries d'oppositions qui concernent des aspects individuels et sociaux distincts et diversifiés qui se sont cristallisés au fil du temps également en termes de variétés.

La perspective cognitive, cependant, dans cette archive, contrairement à l'approche sociale, apporte une préoccupation constante avec la didactisation des théories, travaillant la métalangue des exercices, montrant la manière de transformer le portugais en discipline, proposant des stratégies, des contenus, des activités, des matériaux à utiliser en classe, présentant des modèles, explicitant le faire des actes de lecture et d'écriture et les comportements qui y sont présents à explorer dans les pratiques pédagogiques. Il y aurait là le pouvoir de la norme scolaire dans les processus d' individuation du sujet en rapport à l'État. Un véritable projet d'acculturation par la constitution d'un savoir linguistique spécifiquement scolaire.

\footnotetext{
Quand il en va aussi ouvertement des implications du théorique dans les pratiques, quel sens y a-t-il à vouloir se limiter à un débat "strictement scientifique ", à l'abri du politique, si ce n'est, précisément, pour des raisons en définitive politiques ? (GADET; HAROCHE; HENRY; PÊCHEUX,
}

23 "présupposition que la fonction de l'école, dans le domaine du langage, est d'introduire l'enfant dans le monde de l'écriture, ce qui le fait un citoyen fonctionnellement lettré » (KATO, 1986, p. 7).

24 «à la compréhension du " caractère politico-idéologique d'usage et d'enseignement de la langue à l'école» (SOARES, 1986, p. 7) . 
Observer comment certaines relations entre savoirs savants et savoirs enseignées se construisent et se propagent massivement ne signifie pas ignorer les avancées théoriques et pratiques même dans un cadre réformiste. Au contraire. Cela nous permet de comprendre la situation actuelle dans laquelle certaines oppositions se sont stabilisées de manière simpliste et polarisée, mais qui ne sont pas dépourvus d'une dimension politique et idéologique. Et comprendre que certaines questions comme les tensions qu'il y a entre la langue et les langues comme objet réel et objet d'étude et d'enseignement, doivent être problématisées dans la formation des enseignants.

\section{Références}

AUROUX, Sylvain; WEIL, Yvonne. Dictionnaire des auteurs et des thèmes de la philosophie. Paris: Hachette Education, 1991.

BRASIL. Parâmetros Curriculares Nacionais: terceiro e quarto ciclos do ensino fundamental: língua portuguesa. Brasília: Mec/Inep, 1998.

Seminário Aprendizagem da língua materna: uma abordagem interdisciplinar. Brasília: Instituto Nacional de Estudos e Pesquisas Educacionais, 1983.

III Plano Setorial de Educação, Cultura e Desporto: 19801985. Brasília: Mec, 1980.

CHISS, Jean-Louis; PUECH, Christian. Le langage et ses disciplines XIXe - XXe siècles. Paris, Bruxelles: Ducolot, 1999.

CURY, Carlos Roberto Jamil. Cidadania republicana e educaçáo: Governo Provisório do Mal. Deodoro e Congresso Constituinte de 1890-1891. Rio de Janeiro: DP\&A, 2001. 
GADET, Françoise; HAROCHE, Claudine; HENRY, Paul; PÊCHEUX, Michel. Note sur la question du langage et du symbolique en psychologie. Fundamenta Scientiae, Paris, vol. 3. n. 2, p. 149-159, 1982.

GADET, Francoise ; PÊCHEUX, Michel. La langue introuvable. Paris, Maspero, 1981.

KATO, Mary. No mundo da escrita: uma perspectiva psicolinguística. Série Fundamentos. São Paulo: Ática, 1986.

ORLANDI, Eni. "Ética e política linguística". Línguas e instrumentos linguísticos, n. 1, p. 7-16. Campinas, SP: Pontes, 1998.

PÊCHEUX, Michel; GADET, Françoise. Y-a-t-il une voie pour la linguistique hors du logicisme et du sociologisme . Equivalences, n. 2-3, p. 133-146, 1977.

PÊCHEUX, Michel. Les verités de la palice : linguistique, sémantique, philosophie. Paris: Maspero, 1975.

Analyse automatique du discours. Sciences du comportement. Dupont, 1969.

SILVA, Mariza Vieira da. Manuais escolares e saberes linguísticos. BSEHL Boletin de la Sociedad Española de Historiografia Linguística, n. 11. p. 209224, 2017. Disponível em: <http://www.sehl.es/uacuteltimo-nuacutemero. html>. Acesso em: 01 ago. 2018.

. "Le mouvement et les déplacements de la disciplinarisation des savoirs linguistiques au Brésil : historicité, sujet et société contemporaine". Dossiers HEL : La disciplinarisation des savoirs linguistiques: histoire et épistémologie, n. 5, p. 1-9. 2012. Disponível em: <http://htl.linguist. univ-paris-diderot.fr/hel/dossiers/numero5>. Acesso em: 01 ago. 2018.

SOARES, Magda. Linguagem e escola: uma perspectiva social. Série 
Fundamentos. São Paulo: Ática, 1986.

DOI - http://dx.doi.org/10.5902/2179219436586

fragmentum, n. Especial, Jul./Dez. 2018. 